\title{
Walks on Apollonian networks
}

\author{
Zi-Gang Huang ${ }^{1}$, Xin-Jian $\mathrm{Xu}^{2}$, Zhi-Xi Wu ${ }^{1}$, and Ying-Hai Wang ${ }^{1, *}$ \\ ${ }^{1}$ Institute of Theoretical Physics, Lanzhou University, Lanzhou Gansu 730000, China \\ ${ }^{2}$ Department of Electronic Engineering, City University of Hong Kong, Kowloon, Hong Kong, China
}

(Dated: May 10, 2018)

\begin{abstract}
We carry out comparative studies of random walks on deterministic Apollonian networks (DANs) and random Apollonian networks (RANs). We perform computer simulations for the mean first passage time, the average return time, the mean-square displacement, and the network coverage for unrestricted random walk. The diffusions both on DANs and RANs are proved to be sublinear. The search efficiency for walks with various strategies and the influence of the topology of underlying networks on the dynamics of walks are discussed. Contrary to one's intuition, it is shown that the self-avoiding random walk, which has been verified as an optimal strategy for searching on scale-free and small-world networks, is not the best strategy for the DAN in the thermodynamic limit.

PACS numbers: 89.75.Hc, 05.40.Fb, 89.75.Fb
\end{abstract}

In the past few years, much attention has been devoted to the characterization and modelling of a wide range of complex systems that can be described as networks [1, 2, 3]. The topological properties of real-world networks have been studied extensively. But an even more intriguing task, and a natural extension of these studies, is to understand how the topological structure of networks affects dynamics taking place on top of them [4]. Many dynamic processes have been studied on complex networks, such as epidemic spreading [5], percolation [6], and synchronization [7] et al. These researches show that topologies of networks play a crucial role in determining the system dynamical features.

Random walk has been used for modelling various dynamics in physical, biological, and social contexts [8]. It could also be a mechanism of transport and search on network [9, 10, 11]. Thus, one interesting problem is to study the dynamical behavior of a random walker on networks with different topological properties. Much is known about random walks on both regular and random networks [12, 13]. Recently, there have also been several studies of random walks on small-world networks (SWNs) [14, 15, 16, 17] and on scale-free networks (SFNs) [9, 18, 19, 20]. The impacts of the heterogeneous topological structures of the networks on the nature of the diffusive and relaxation dynamics of the random walk were probed [9, 10, 21, 22]. Also, finding efficient algorithms for searching on networks with different topological properties is an important issue related to random walks [9, 18, 20].

In this paper we investigate walking processes taking place upon the determinist Apollonian network (DAN) [23] and its variation, the random Apollonian network (RAN) [24]. The DAN can be defined based of the ancient problem of filling space with spheres, first tackled by the Greek mathematician Apollonius of Perga [25]. That is, starting with an initial array of touching disks, which have curvilinear-triangle interstices, disks are added inside each existed interstice in the present configuration, such that these disks touch each of the disks

*For correspondence: yhwang@1zu.edu.cn bounding the curvilinear triangles. These added disks give rise to three smaller interstices, which will be filled in the next generation. This process is then repeated for successive generations. The DAN is constructed based on this process by considering each disk as a node, and the disks in contact as the corresponding nodes connected. For each new node added to a certain triangle (corresponding to the curvilineartriangle interstice), the three vertices are linked to, and three new triangles are created in the network, into which nodes will be inserted in the next generation. Different from recursive constructing of the DAN [23], the RAN starts with a triangle containing three nodes. Then, at each time step, one triangle is randomly selected to add a new node [24]. Both networks are simultaneously scale-free, small-world, Euclidean, and space filling, and have attracted an increasing interest recently [26, 27, 28].

We carry out the random walk along the bonds of a given network as follows: (i) There is only one walker on the network at a time; (ii) The random walker is injected onto a randomly chosen node on the network, a new node for each walker. We will call this node the "origin" of the walk; (iii) At each discrete time step $t$, the walker will jump to one nearest neighbor of its current node according to one certain strategy; (iv) We average over different random walkers and realizations of the network until the results converge.

The walk strategies adopted by the walker include the following: random walk (RW), no-back (NB) walk, no-triangleloop (NTL) walk, no-quadrangle-loop (NQL) walk, and selfavoiding (SA) random walk. For the RW, the walker may unrestrictedly jump to a neighbor node by randomly taking one of the links. It forgets all information about its past. The NB walk implies that a random walker, if possible, will not return to the node it was situated at the previous step. Similarly, NTL and NQL random walks mean that the walker will try to avoid walking in loops, with three or four edges, respectively, unless there is no other choice. We mention that the NQL walk also includes the NTL, which means it eliminates quadrangle loops as well as triangle loops. Finally, the SA random walk implies that the walker is the smarter. It tries to avoid revisiting the node that it has already visited in a run of the search. Surely, the SA walk also includes the NTL and NQL walks. 

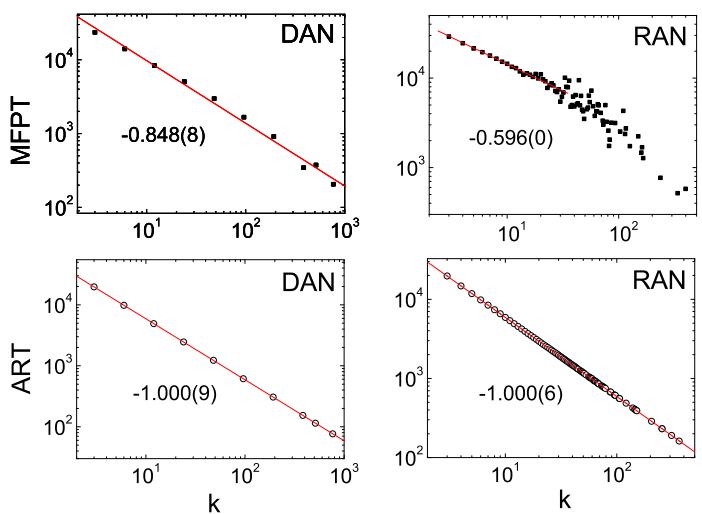

FIG. 1: (Color online) The mean first passage times $\left\langle T_{i j}\right\rangle$ (upper panels) and the average return time $\left\langle T_{i i}\right\rangle$ (lower panels), both of which are average over the results of given $k$ 's, for DANs (left-hand) and RANs (right-hand). Fitted linear relations of average return time (solid lines) are obtained with a slope of -1 for both DANs and RANs.

One can imagine that an unrestricted RW walker may be trapped into high-clustering regions and revisit nodes there for a long time by reason of walking in loops. While a walker adopting other strategies like NB, NTL and NQL can escape from those regions easier, and therefore improve the search efficiency. Thus, different clustering properties of the underlying networks will induce different efficiencies of the above mentioned strategies. In return, the clustering effect of the networks could be reflected by the efficiencies of those search strategies.

In the context of transport and search, the mean first passage time (MFPT) and the average return time (ART) are important characteristics of the random walk. The average return time denoted by $\left\langle T_{i i}\right\rangle$ is the average time needed for a walker to return to the origin $i$, which is a special case of MFPT from $i$ to $j$ denoted by $\left\langle T_{i j}\right\rangle$. Noh and Rieger [18] have proved that the MFPT is negatively correlated with $K_{j}$, and $\left\langle T_{i i}\right\rangle$ is determined only by the total number of links and the degree of the node $i$

$$
\left\langle T_{i i}\right\rangle=\mathcal{N} / K_{i}
$$

with $\mathcal{N}=\sum_{j} K_{j}(j=1, \ldots, N)$. Namely, nodes with higher degrees are visited earlier and more frequently, and therefore targets on these nodes are more easily found out than on nodes with smaller degrees. In Fig. 11we depict simulations of the MFPT (the upper panels) and ART (the lower panels) as a function of nodes degree for the DAN and RAN with size $N=9844$. After averaging over the MFPT and ART for a given degree $k$, the linear property of the log-log plot of ART (the lower panels) is in excellent agreement with Eq. (1), and the negative correlation between MFPT and $k$ are also presented (the upper panels).

In the following, we will investigate walking processes on DANs and RANs. The corresponding results on WattsStrogatz (WS) $\left(K=3, p_{0}=0.1\right)$ [29] and Barabási-Albert
TABLE I: The PSP threshold $P_{c}$ and average path length $\langle L\rangle$ of the four networks with size $N=9844$.

\begin{tabular}{lrrrr}
\hline \hline & DAN & RAN & BA & WS \\
\hline$P_{c} \approx$ & $4 / N$ & 0.03 & 0.3 & 0.63 \\
$\langle L\rangle$ & $4.06(5)$ & $5.40(9)$ & $4.27(9)$ & $8.61(9)$ \\
\hline \hline
\end{tabular}

(BA) $\left(m=m_{0}=3\right.$ ) [30] networks are also presented for comparison. Note that, the four networks have the same average degree $\langle k\rangle=6$.

Firstly, let us discuss the search efficiency of all the five walk strategies mentioned above for the four networks. We suppose at every step, the walker adopting various strategies only know neighbors of its present node. So if the target is at one neighbor of the present node where the walker stays, this round of search is over. The walks for the five strategies are performed and the search times (see Fig. 2) are averaged over 200 different couples of initial positions and targets of the walker, and 50 realizations of the networks. One can find some common properties from Fig. 2] For example, the approximate power-law relations hold for all search processes of RW, NB, NTL, NQL and SA walks. As another example, the RW walk is the most inefficient search strategy for all the four networks. Nevertheless, some obvious differences still exist among them. The search efficiencies of NB, NTL and NQL walks nearly collapse into one only for BA network (see Fig. 22 (c), also in Ref. [20]). This can be understood by its comparatively smallest clustering coefficient as to the other three networks. In the weak clustering networks, the NB, NTL and NQL walkers, who adopt increasingly strict law to avoid revisit, can not take their advantages and their search efficiencies keep very closely or even collapse into one. Hence, for DANs and RANs, the efficiency-improvement of NTL and NQL walks as to the NB walk reflects the fact that high clustered nodes are popular on them.

The SA walk was proved to be generally the most efficient strategy for searching on networks, whose small-world properties were considered to be the reason [20]. However, it is counterexample for the DAN (see Fig. 2(b)). The DAN is one of typical regular networks which has deterministic size when the number of the generations is certain [23, 26]. Its short average length and high clustering coefficient indeed describe a small-world scenario. Fig. 2] b) shows the average search times for DANs with 3 to 8 generations, corresponding to network sizes $16,43,124,367,1096$, and 3283, respectively. Contrast to the conclusions in Ref. [20], the SA walk does not reduce the search time and is even more inefficient than the NTL and NQL walks when $N$ is large. We argue that what is important for the improvement of the search efficiency is not taking more strict law to eliminate repeating visits, but considering the factual structure of the networks.

According to the former analysis of MFPT, we know that the high-degree nodes would be visited early, so as to be avoided by SA walker preferentially. Thus, to some extent the SA search process can be considered as the question of intentional attack [31] or preferential site percolation (PSP) on networks. The intentional attack means the removal of nodes and 

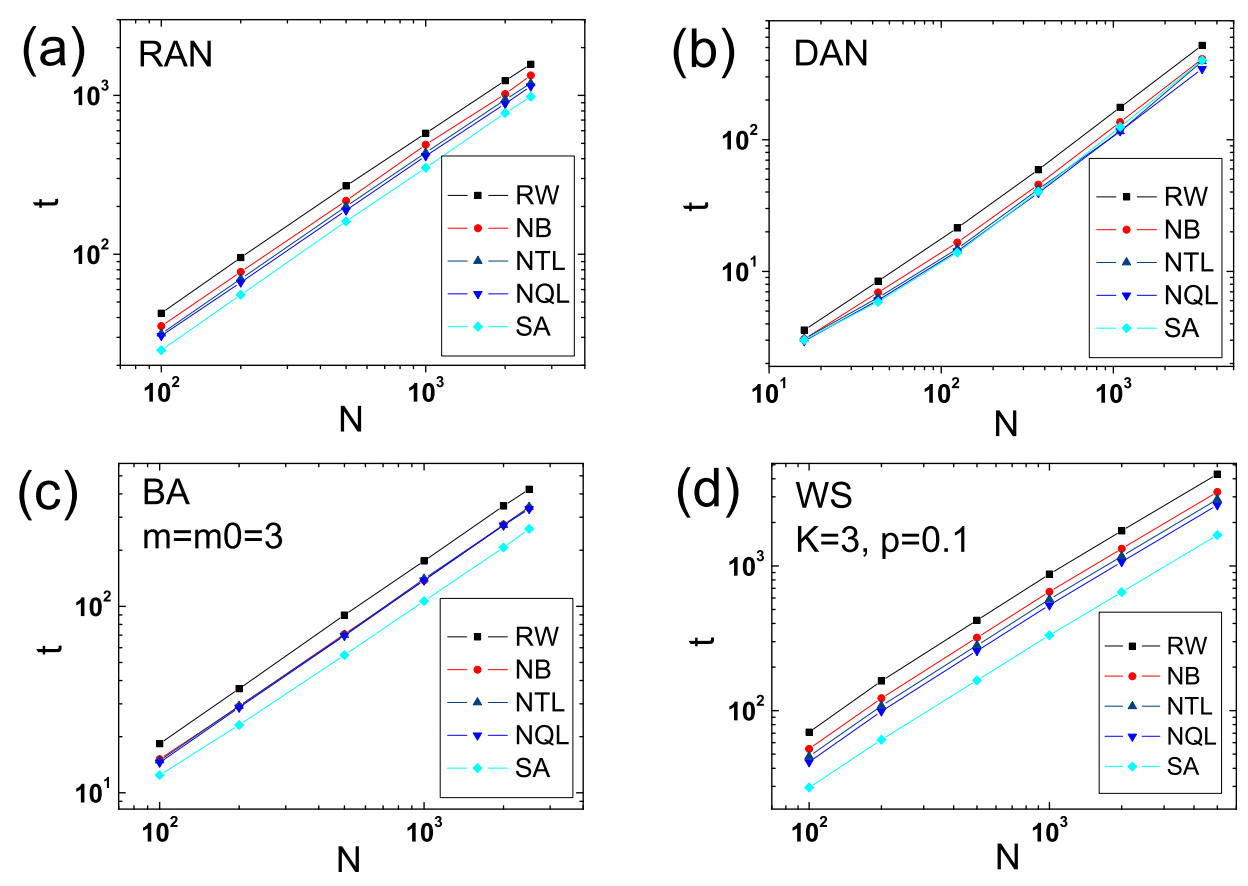

FIG. 2: (Color online) Average search times of various search strategies for RANs (a), DANs (b), BA networks (c), and WS networks (d).

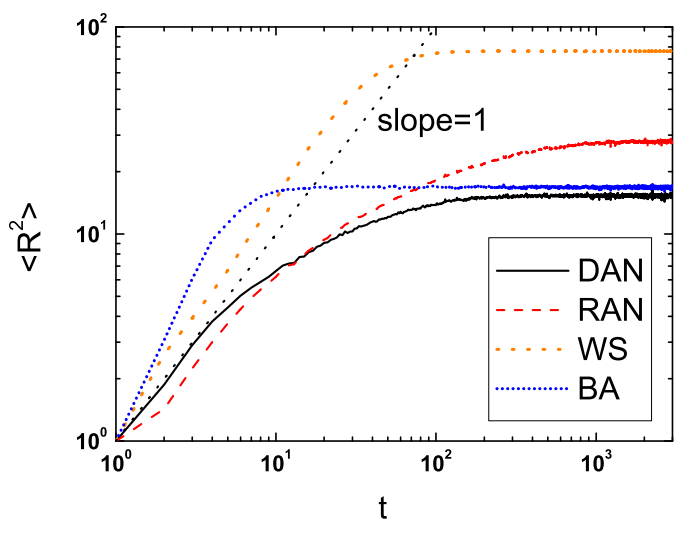

FIG. 3: Mean-square distance $\left\langle R^{2}\right\rangle$ as a function of time for DANs, RANs, WS and BA networks with size $N=9844$ and average degree $\langle k\rangle=6$ fixed. The dot line with slope 1 is plotted for comparison.

their incident edges aiming at those greatest degree nodes, and the PSP threshold $P_{c}$ is a measure of how robust the network is against this attack. When the network size is relatively small $\left(N<10^{5}\right)$, DANs and RANs are much frailer than SW and SF networks under the intentional attacks (see Table. Ifor $P_{c}$ ),

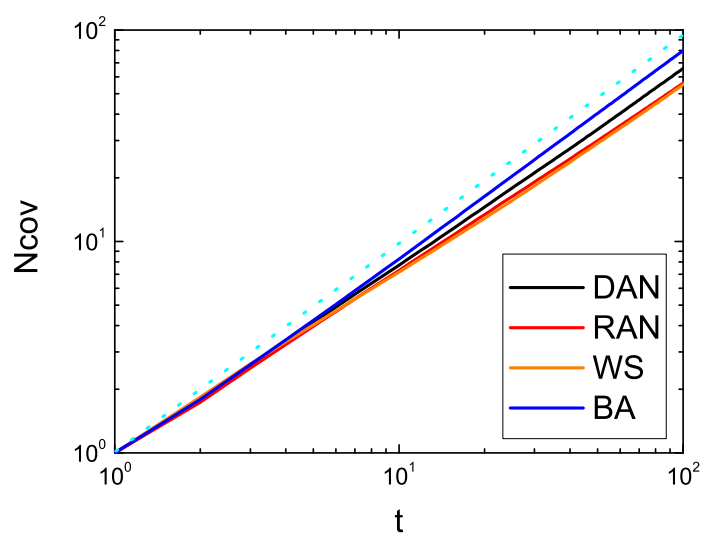

FIG. 4: (Color online) Network coverage $N_{c o v}$ after $t$ steps on (solid line from top to bottom) BA networks, DANs, RANs and WS networks with $N=9844$ and $\langle k\rangle=6$ fixed.

because of the crucial importance of the high-degree nodes to the network integrates [24]. When walking on networks, if the walkers strictly avoid visiting these crucial high-degree nodes, 
they can hardly move from their current part of the network to other parts, as if the network was intentionally attacked and disintegrated into partes. However, as a search strategy, the SA search processes on the original network are not totally the same as the intentional attacks, because SA walker at node $i$ can revisit nodes, including these crucial nodes, although, with probability $1 / k_{i}$ only in condition that all the $k_{i}$ neighbors have already been visited. The compulsive revisits insure that the targets in different parts of networks can finally be found. Considering the similarity between SA search and intentional attack or PSP, we can conclude that the SA search strategy, compared with other mentioned strategies, will improve the search efficiency to certain extent for the networks with higher PSP threshold.

Next, we will present our simulation results for two quantities, the mean-square displacement and the network coverage (the average number of distinct sites visited). The meansquare displacement $\left\langle R^{2}(t)\right\rangle$ of a particle diffusing in a given space, which is a measure of the distance $R$ covered by a typical RW walker after performing $t$ steps, is one of the most basic quantities in the random walk theory [16, 19]. In most cases, this quantity is described by an expression of the form $\left\langle R^{2}(t)\right\rangle \sim t^{a}$. The value of the parameter $a$ classifies the type of diffusion into normal linear diffusion $(a=1)$, subdiffusion $(a>1)$, or superlinear diffusion $(a<1)$. When considering the unrestricted RW walk along the bonds of networks, the maximum value of $a$ can be 2 [19]. To calculate $\left\langle R^{2}(t)\right\rangle$ we first, at each time step, find the minimal distance from the current position of the walker to the origin (i.e., the smallest number of steps needed for the walker to reach the origin) using a breadth-first search method. Then we allow the walker to move through the network until $\left\langle R^{2}(t)\right\rangle$ has saturated. Finally, the results are average over different initial positions of the walkers and realizations of the network. We simulate $\left\langle R^{2}(t)\right\rangle$ for the DAN, RAN, WS and BA networks with $N=9844$, and report it as a function of time in Fig. 3 One important feature is the fact that $\left\langle R^{2}(t)\right\rangle$ equilibrates after a few steps to a constant displacement value. This is a simple manifestation of the small diameter of these finite networks. Note also that, because of the differences of the average path length $\langle L\rangle$ of these networks (see Table. I for $\langle L\rangle$ ), the plateau values are also different. For the DANs and RANs, one can find that the slope of $\left\langle R^{2}\right\rangle$ in the early time is small, especially that of RANs. This can be explained as a result of their highly clustering effect which induces the walker initially spends many time exploring the cluster it was created in and thus the distance to the origin increases slowly. After a few steps the walker escapes its initial territory and diffuses around the entire network. From the slope of $\left\langle R^{2}\right\rangle$, we know that diffusions both on DANs and RANs are sublinear, and diffusions both on BA and WS networks are superlinear. The network coverage denoted by $N_{\text {cov }}$ is defined as average number of distinct visited nodes of the RW walker. Results of $N_{\text {cov }}$ on the four networks are presented in Fig. 4. The number of steps performed is nearly two orders of magnitude smaller than the size of the networks, in order to decrease finite size effects. The clustering effects of DANs and RANs are reflected by their relatively low coverage compared to BA networks.

In summary, we present comparative studies of the dynamics of random walks on deterministic Apollonian networks and random Apollonian networks with other networks. The efficiency of search strategies are simulated and the clustering and short path effects of networks to the dynamics are discussed. It is found that, for the DAN with large size, the best search strategy is no longer the self-avoiding random walk. The importance of the high-degree nodes to the network integrates are suppose to be one reason. Thus the search strategy on networks should be assigned closely based on the topological structure of the underlying networks, including the clustering, the average path length as well as the essentiality of the high-degree nodes. Since search is a problem of extreme importance for so many natural and artificial networks, this finding may be valuable in practicability. Finally, the simulation results of mean-square displacement $\left\langle R^{2}(t)\right\rangle$ and network coverage $N_{\text {cov }}$ also show the influences of the structure of networks to the dynamics of random walks.
[1] R. Albert and A.L. Barabási, Rev. Mod. Phys. 74, 47 (2002).

[2] S.N. Dorogovtsev and J.F.F. Mendes, Adv. Phys. 51, 1079 (2002).

[3] M.E.J. Newman, SIAM Rev. 45, 167 (2003).

[4] S.H. Strogatz, Nature (London) 410, 268 (2001).

[5] R. Pastor-Satorras and A. Vespignani, Phys. Rev. Lett. 86, 3200 (2001); R. Pastor-Satorras and A. Vespignani, Phys. Rev. E 63, 066117 (2001); M.E.J. Newman, ibid 66, 016128 (2002).

[6] D.S. Callaway, M.E.J. Newman, S.H. Strogatz, and D.J. Watts, Phys. Rev. Lett. 85, 5468 (2000); M.E.J. Newman, S.H. Strogatz, and D.J. Watts, Phys. Rev. E 64, 026118 (2001); R. Cohen, D. ben-Avraham, and S. Havlin, ibid 66, 036113 (2002).

[7] M. Barahona and L.M. Pecora, Phys. Rev. Lett. 89, 054101 (2002); T. Nishikawa, A.E. Motter, Y.-C. Lai, and F.C. Hoppensteadt, ibid 91, 014101 (2003); H. Hong, B.J. Kim, M.Y. Choi, and H. Park, Phys. Rev. E 69, 067105 (2004).

[8] F. Spitzer, Principles of Random Walk, 2nd ed. (Springer-
Verlag, New York, 1976).

[9] L.A. Adamic, R.M. Lukose, A.R. Puniyani, and B.A. Huberman, Phys. Rev. E 64, 046135 (2001).

[10] R. Guimerá, A. Díaz-Guilera, F. Vega-Redondo, A. Cabrales, and A. Arenas, Phys. Rev. Lett. 89, 248701 (2002).

[11] P. Holme, Advances in Complex Systems 6, 163 (2003).

[12] M.N. Barber and B.W. Ninham, Random and Restricted Walks (Gordon and Breach, New York, 1970).

[13] B.D. Hughes, Random Walks and Random Environments (Clarendon, Oxford, 1996), Vols. 1 and 2.

[14] S.A. Pandit and R.E. Amritkar, Phys. Rev. E 63, 041104 (2001).

[15] J. Lahtinen, J. Kertsz, and K. Kaski, Phys. Rev. E 64, 057105 (2001).

[16] E. Almaas, R.V. Kulkarni, and D. Stroud, Phys. Rev. E 68, 056105 (2003).

[17] P.E. Parris and V.M. kenkre, Phys. Rev. E 72, 056119 (2005).

[18] J.D. Noh and H. Rieger, Phys. Rev. Lett 92, 118701 (2004); 
Phys. Rev. E 69, 036111 (2004); cond-mat/0509564

[19] L.K. Gallos, Phys. Rev. E 70, 046116 (2004).

[20] S.-J. Yang, Phys. Rev. E 71, 016107 (2005).

[21] S. Jespersen, I.M. Sokolov, and A. Blumen, Phys. Rev. E 62, 4405 (2000);

[22] B. Tadić, Eur. Phys. J. B 23, 221 (2001).

[23] J.S. Andrade, Jr., H.J. Herrmann, R.F.S. Andrade, and L.R. da Silva, Phys. Rev. Lett. 94, 018702 (2005)

[24] T. Zhou, G. Yan, and B.-H. Wang, Phys. Rev. E 71, 046141 (2005).

[25] D.W. Boyd, Can. J. Math. 25, 303 (1973).

[26] J.P.K. Doye and C.P. Massen, Phys. Rev. E 71, 016128 (2005).
[27] R.F.S. Andrade and H.J. Herrmann, Phys. Rev. E 71, 056131 (2005).

[28] Z. Zhang, L. Rong, and F. Comellas, cond-mat/0502591 Z. Zhang, F. Comellas, G. Fertin, and L. Rong, cond-mat/0503316

[29] D.J. Watts and S.H. Strogatz, Nature 393, 440 (1998).

[30] A.-L. Barabási and R. Albert, Science 286, 509 (1999); A.-L. Barabási, R. Albert, and H. Jeong, Physica A 272, 173 (1999).

[31] R. Albert, H. Jeong, and A.-L. Barabási, Nature (London) 406, 378 (2000). 